\title{
REMARKS ON THE TWO AND THREE MEMBRANES PROBLEM
}

\author{
ASSIS AZEVEDO \\ Department of Mathematics, \\ University of Minho, \\ Campus de Gualtar, \\ 4710-057 Braga,$$
\text { Portugal }
$$ \\ E-mail: assis@math.uminho.pt
JOSÉ-FRANCISCO RODRIGUES*
CMUC-University of Coimbra, University of Lisbon/CMAF,
Av. Prof. Gama Pinto, 2, 1649-003 Lisbon,
Portugal
E-mail: rodrigue@fc.ul.pt \\ LISA SANTOS ${ }^{\dagger}$ \\ CMAF-University of Lisbon, \\ Department of Mathematics, \\ University of Minho, \\ Campus de Gualtar, \\ 4710-057 Braga, \\ Portugal \\ E-mail: lisa@math.uminho.pt
}

We consider the problem of finding the equilibrium position of two or three membranes constrained not to pass through each other. For general linear second order elliptic operators with measurable coefficients we prove the Lewy-Stampacchia type inequalities and we establish sufficient conditions on the external forces to obtain the stability of the coincidence sets of the membranes, in analogy with the obstacle problem. 


\section{Introduction and notations}

Among the manifold of unilateral problems that can be formulated as an elliptic variational inequality (see $[4,5]$ or $[6]$ )

$$
U \in \mathbb{K}: \quad\langle A U-F, V-U\rangle \geq 0, \quad \forall V \in \mathbb{K},
$$

we shall consider here some new remarks on the $N$-membranes problem, illustrated in the cases $N=2$ and $N=3$ by their analogies with the one and two obstacle problems, respectively.

In (1) $U=\left(u_{1}, \ldots, u_{n}\right)$ may represent the equilibrium displacements of $N$ elastic membranes minimizing the energy functional (here $\nabla u=$ $\left(u_{x_{1}}, \ldots, u_{x_{N}}\right)$ denotes the gradient)

$$
E\left(u_{1}, \ldots, u_{n}\right)=\sum_{k=1}^{N} \int_{\Omega}\left(\frac{1}{2}\left|\nabla u_{k}\right|^{2}-f_{k} u_{k}\right) d x,
$$

subject to external forces $F=\left(f_{1}, \ldots, f_{N}\right)$, in the convex set

$$
\begin{gathered}
\mathbb{K}_{N}=\left\{\left(v_{1}, \ldots, v_{n}\right) \in\left[H^{1}(\Omega)\right]^{N}: v_{1} \geq \cdots \geq v_{N} \text { a.e. in } \Omega,\right. \\
\left.v_{k}=\beta_{k} \text { on } \partial \Omega, k=1, \ldots, N\right\},
\end{gathered}
$$

where $\beta_{1}, \ldots, \beta_{N}$ are given functions $\left(\beta_{1} \geq \cdots \geq \beta_{N}\right)$ describing their rigid supports on the boundary $\partial \Omega$ of a domain $\Omega \subset \mathbb{R}^{n}$ (actually $n=2$ is the physical situation).

This problem was considered in [9] and [10] (see also [4]) for $N=2$, respectively in the linear case and for two surfaces of constant mean curvature. This case is strongly related with the one obstacle problem, which can be also written in the form (1). For instance, when $N=1$ in (2) and, for a given smooth obstacle $\psi=\psi(x), \psi \leq \beta$ on $\partial \Omega$, the associated convex set is given by

$$
\mathbb{K}_{\psi}=\left\{\xi \in H^{1}(\Omega): \xi \geq \psi \text { a.e. in } \Omega, \xi=\beta \text { on } \partial \Omega\right\}
$$

and the operator $A$ in (1) is $-\Delta=-\sum_{k=1}^{N} \frac{\partial^{2}}{\partial x_{k}^{2}}$. For this "lower" obstacle problem, the well-known Lewy-Stampacchia inequalities (see [6] or [8] for instance) can be written in the form

$$
f \leq A u \leq f \vee A \psi \quad \text { a.e. in } \Omega .
$$

Here we use the standard notations $\xi^{+}=\xi \vee 0, \xi^{-}=\xi \wedge 0$,

$$
\bigvee_{k=1}^{2} \xi_{k}=\xi_{1} \vee \xi_{2}=\sup \left\{\xi_{1}, \xi_{2}\right\} \quad \text { and } \quad \bigwedge_{k=1}^{2} \xi_{k}=\xi_{1} \wedge \xi_{2}=\inf \left\{\xi_{1}, \xi_{2}\right\}
$$


If we consider the symmetrical "upper" obstacle problem with

$$
\mathbb{K}^{\varphi}=\left\{\xi \in H^{1}(\Omega): \xi \leq \varphi \text { a.e. in } \Omega, \xi=\beta \text { on } \partial \Omega\right\}
$$

for a given $\varphi=\varphi(x)$, with $\varphi \geq \beta$ on $\partial \Omega$, the corresponding solution $w$ to (1) will satisfy the symmetrical Lewy-Stampacchia inequalities

$$
f \wedge A \varphi \leq A w \leq f \quad \text { a.e. in } \Omega .
$$

The $N$-membrane problem was considered in [2] for $N$ linear coercive elliptic operators of second order, in particular the $\left[W^{2, p}(\Omega)\right]^{N}$ regularity of the solution was shown for $p \geq 2$ by using an iterative penalization technique. For $N=2$ the membranes problem can also be considered, once the solution is known, as two one obstacle problems. While for $N=3$ only the upper and the lower membranes are of this type, the membrane in the middle may be considered solution of a two obstacles problem. This corresponds to a problem of the type (1) with the convex set given in the form

$$
\mathbb{K}_{\psi}^{\varphi}=\mathbb{K}_{\psi} \cap \mathbb{K}^{\varphi}=\left\{\xi \in H^{1}(\Omega): \psi \leq \xi \leq \varphi \text { a.e. in } \Omega, \xi=\beta \text { on } \partial \Omega\right\},
$$

with given obstacles $\psi \leq \varphi$ in $\Omega$ and $\psi \leq \beta \leq \varphi$ on $\partial \Omega$, for which the corresponding Lewy-Stampacchia inequalities for the solution $v$ are given by

$$
f \wedge A \varphi \leq A v \leq f \vee A \psi \quad \text { a.e. in } \Omega .
$$

The iteration of these inequalities yields the new set of $N$ inequalities for the solution $\left(u_{1}, \ldots, u_{N}\right)$ of the $N$-membranes problem

$$
\bigwedge_{k=1}^{l} f_{k} \leq A u_{l} \leq \bigvee_{k=l}^{N} f_{k}, \quad \text { a.e. in } \Omega, \quad l=1, \ldots, N
$$

which allows to reduce the regularity of the solutions to the corresponding regularity of a system of equations, extending, in particular, the result of [2] for certain $p<2$. We obtain here the inequality (7) for a general linear elliptic operator (using the summation convention on repeated indices)

$$
A v=-\left(a_{i j} v_{x_{i}}+e_{j} v\right)_{x_{j}}+b_{i} v_{x_{i}}+c v
$$

where the measurable coefficients $a_{i j}, e_{j}, b_{i}$ and $c$ satisfy the minimum integrability assumptions for obtaining solutions in $H^{1}(\Omega)$ and $A$ satisfies a weak maximum principle. In [1] we extend (7) for nonlinear operators of $p$-Laplacian type. 
Another interesting consequence of (7) is the stability of the coincidence sets (and their intersections)

$$
I_{k}=\left\{x \in \Omega: u_{k}(x)=u_{k+1}(x)\right\}, \quad k=1, \ldots, N-1
$$

where two consecutive membranes touch each other when the external forces vary.

For $N=2$ there is only one possible coincidence set $I_{1}$. In Section 2 we show that if the two forces associated with the two membranes are almost everywhere different in $\Omega\left(f_{1} \neq f_{2}\right.$ a.e. in $\left.\Omega\right)$ then the characteristic function $\chi_{I_{1}}$ of $I_{1}$ converges strongly in $L^{q}(\Omega)$ for variations of the forces in a certain $L^{p_{0}}(\Omega)$. This result combines a remark of [10] with results of [6] and [7] for the obstacle problem, that imply the two equations

$$
A u_{k}-f_{k}=(-1)^{k} \frac{1}{2}\left(f_{1}-f_{2}\right) \chi_{I_{1}} \quad \text { a.e. in } \Omega, \quad k=1,2 .
$$

For $N=3$ there are three possible coincidence sets $I_{1}, I_{2}$ and $I_{1,2}=$ $I_{1} \cap I_{2}$ and, in general, we easily see that the $N$-membranes problem may have $\frac{N(N-1)}{2}$ possible coincidence sets. The extension of (10) to the general $N$-system is possible but becomes then a more complex issue that is also the subject of [1]. Inspite of the Lewy-Stampacchia inequalities (7) and the regularity results being valid for $N>3$, here we consider only the case of three membranes. In Section 3, we give sufficient conditions on the three forces for the stability of the characteristic functions $\chi_{I_{1}}, \chi_{I_{2}}$ and $\chi_{I_{1,2}}$. In addition to the natural requirement of $f_{1} \neq f_{2}$ and $f_{2} \neq f_{3}$ a.e. in $\Omega$ we identify the somehow two new unexpected conditions for the stability of $\chi_{I_{1,2}}=\chi_{I_{1}} \chi_{I_{2}}$ :

$$
f_{1} \neq \frac{f_{2}+f_{3}}{2}, \quad \text { and } \quad f_{3} \neq \frac{f_{1}+f_{2}}{2} \quad \text { a.e. in } \Omega .
$$

In this work we shall impose the following assumptions $(\nu>0)$ :

$$
\begin{gathered}
a_{i j} \in L^{\infty}(\Omega), \quad a_{i j} \xi_{i} \xi_{j} \geq \nu|\xi|^{2} \quad \text { a.e. in } \Omega \quad \forall \xi \in \mathbb{R}^{n} \\
b_{i}, e_{i} \in L^{p}(\Omega), \quad c \in L^{p / 2}(\Omega), \quad p>n=2 \text { or } p=n \geq 3 \\
c-\left(e_{i}\right)_{x_{i}} \geq 0 \quad \text { in } \mathcal{D}^{\prime}(\Omega) \\
f_{k} \in L^{p_{0}}(\Omega), \quad p_{0}>1 \text { if } n=2 \quad \text { or } \quad p_{0} \geq \frac{2 n}{n+2} \text { if } n \geq 3 \\
\beta_{k} \in H^{\frac{1}{2}}(\partial \Omega), \quad \beta_{1} \geq \cdots \geq \beta_{N} \text { a.e. in } \partial \Omega
\end{gathered}
$$


for $k=1, \ldots, N$, for $N=2$ and $N=3$.

Here $\Omega$ is a bounded open subset of $\mathbb{R}^{n}$ with a Lipschitz boundary, so that $H^{\frac{1}{2}}(\partial \Omega)$ is the trace space of functions in $H^{1}(\Omega)$, which by Sobolev theorem is imbedded in $L^{s}(\Omega)$ with arbitrary $s<\infty$ if $n=2$ and $s \leq \frac{2 n}{n-2}$ if $n \geq 3$.

Under the above assumptions, we may define

$$
\langle A U-F, V\rangle=\sum_{k=1}^{N}\left[a\left(u_{k}, v_{k}\right)-\int_{\Omega} f_{k} v_{k}\right]
$$

for arbitrary $U=\left(u_{1}, \ldots, u_{N}\right), V=\left(v_{1}, \ldots, v_{N}\right) \in\left[H^{1}(\Omega)\right]^{N}$. Here

$$
\langle A u, v\rangle=a(u, v)=\int_{\Omega}\left(a_{i j} u_{x_{i}}+e_{j} u\right) v_{x_{j}}+\int_{\Omega} b_{i} u_{x_{i}} v+\int_{\Omega} c u v
$$

defines the elliptic operator (8) that, under assumptions (11)-(13), even when is not coercive, it satisfies a maximum principle, which is a consequence of the following Lemma that we shall use (see [6] §4.7):

Lemma 1.1. If $v \in H_{0}^{1}(\Omega)$ is such that

$$
a\left(v,(v-s)^{+}\right) \leq 0 \quad \forall s, 0<s<\infty,
$$

then we must have $v \leq 0$ a.e. in $\Omega$.

\section{The $N=2$ membranes problem}

In this section we set $u_{1}=u, u_{2}=v, f_{1}=f$ and $f_{2}=g$, so that, with $N=2$ in (3), the 2-membranes problem corresponds to find $(u, v) \in \mathbb{K}_{2}$ such that

$$
a(u, \xi-u)+a(v, \eta-v) \geq \int_{\Omega} f(\xi-u)+\int_{\Omega} g(\eta-v), \quad \forall(\xi, \eta) \in \mathbb{K}_{2}(18)
$$

We can approximate its solution when $\varepsilon \rightarrow 0$ by the solution $\left(u_{\varepsilon}, v_{\varepsilon}\right)$ defined by the system, similarly to [10]

$$
\left\{\begin{array}{l}
A u_{\varepsilon}+\varphi \theta_{\varepsilon}\left(u_{\varepsilon}-v_{\varepsilon}\right)=f \\
A v_{\varepsilon}-\varphi \theta_{\varepsilon}\left(u_{\varepsilon}-v_{\varepsilon}\right)=g
\end{array} \quad \text { in } \Omega\right.
$$

with boundary conditions $u_{\varepsilon}=\beta_{1}$ and $v_{\varepsilon}=\beta_{2}$ on $\partial \Omega$, where for each $\varepsilon>0$, $\theta_{\varepsilon}: \mathbb{R} \longrightarrow \mathbb{R}$ is a smooth nondecreasing function such that

$$
\theta_{\varepsilon}(s)=\left\{\begin{aligned}
0 & \text { if } s \geq 0, \\
-1 & \text { if } s \leq-\varepsilon
\end{aligned}\right.
$$


and $\varphi=\frac{1}{2}(g-f)^{+} \in L^{p_{0}}(\Omega)$, by assumption (14).

Proposition 2.1. There exists a unique solution $\left(u_{\varepsilon}, v_{\varepsilon}\right) \in\left[H^{1}(\Omega)\right]^{2}$ to (19) with the given Dirichlet data and a constant $C>0$ independent of $\varepsilon>0$ such that

$$
\left\|u_{\varepsilon}\right\|_{H^{1}(\Omega)}+\left\|v_{\varepsilon}\right\|_{H^{1}(\Omega)} \leq C .
$$

Moreover,

$$
v_{\varepsilon} \leq u_{\varepsilon}+\varepsilon \quad \text { a.e. in } \Omega \text {. }
$$

Proof: Since $\left|\theta_{\varepsilon}\right| \leq 1$, the existence may be shown as a consequence of the "a priori" estimate (21), which can be obtained as in pages 120-122 of [6]. The uniqueness is a consequence of the monotonicity of $\theta_{\varepsilon}$ and the weak maximum principle for $A$.

To show (22), take $s>0$ and consider $\xi=v_{\varepsilon}-u_{\varepsilon}-\varepsilon$. By (15) we have $\xi^{+}$and $(\xi-s)^{+}$belong to $H_{0}^{1}(\Omega)$. Using (13) and the variational formulation of (19), we obtain

$$
\begin{aligned}
a\left(\xi,(\xi-s)^{+}\right) & =\left\langle A\left(v_{\varepsilon}-u_{\varepsilon}\right),(\xi-s)^{+}\right\rangle-\varepsilon \int_{\Omega}\left(e_{i}(\xi-s)_{x_{i}}^{+}+c(\xi-s)^{+}\right) \\
& \leq\left\langle A\left(v_{\varepsilon}-u_{\varepsilon}\right),(\xi-s)^{+}\right\rangle=\int_{\Omega}\left(g-f+2 \varphi \theta_{\varepsilon}\left(u_{\varepsilon}-v_{\varepsilon}\right)\right)(\xi-s)^{+} \\
& \leq \int_{\left\{v_{\varepsilon}>u_{\varepsilon}+\varepsilon+s\right\}}(g-f+2 \varphi)\left(v_{\varepsilon}-u_{\varepsilon}-\varepsilon-s\right)^{+} \\
& \leq 0
\end{aligned}
$$

since $2 \varphi=(g-f)^{+} \geq g-f$ and $\theta_{\varepsilon}\left(u_{\varepsilon}-v_{\varepsilon}\right)=-1$ in $\left\{v_{\varepsilon}>u_{\varepsilon}+\varepsilon+s\right\}$.

Hence

$$
a\left(\xi^{+},\left(\xi^{+}-s\right)^{+}\right)=a\left(\xi,(\xi-s)^{+}\right) \leq 0, \quad \forall s>0
$$

and, by Lemma 1.1, we conclude that $\xi^{+}=\left(v_{\varepsilon}-u_{\varepsilon}-\varepsilon\right)^{+} \leq 0$ and $(22)$ follows.

Theorem 2.1. When $\varepsilon \rightarrow 0$ the solutions $\left(u_{\varepsilon}, v_{\varepsilon}\right)$ of (19) satisfy

$$
\left(u_{\varepsilon}, v_{\varepsilon}\right) \longrightarrow(u, v) \quad \text { in }\left[H^{1}(\Omega)\right]^{2}-\text { weak, }
$$

where $(u, v) \in \mathbb{K}_{2}$ is the unique solution to (18). In addition,

$$
f \leq A u \leq f \vee g, \quad f \wedge g \leq A v \leq g \quad \text { hold a.e. in } \Omega .
$$


Proof: The estimate (21) implies the existence of at least a subsequence satisfying (23) in $\left[H^{1}(\Omega)\right]^{2}$-weak and $\left[L^{2}(\Omega)\right]^{2}$-strong, by compactness. Hence the limit $(u, v)$, by $(22)$, must be in $\mathbb{K}_{2}$. For any $(\xi, \eta) \in \mathbb{K}_{2}$, multiplying the first equation of (19) by $\xi-u_{\varepsilon}$ and the second one by $\eta-v_{\varepsilon}$, we get

$$
a\left(u_{\varepsilon}, \xi-u_{\varepsilon}\right)+a\left(v_{\varepsilon}, \eta-v_{\varepsilon}\right)+\Phi_{\varepsilon}=\int_{\Omega} f\left(\xi-u_{\varepsilon}\right)+g\left(\eta-v_{\varepsilon}\right),
$$

where

$$
\Phi_{\varepsilon}=\int_{\Omega} \varphi \theta_{\varepsilon}\left(u_{\varepsilon}-v_{\varepsilon}\right)\left[(\xi-\eta)-\left(u_{\varepsilon}-v_{\varepsilon}\right)\right] \leq 0 .
$$

Taking the $\liminf _{\varepsilon \rightarrow 0}$ in

$$
a\left(u_{\varepsilon}, \xi-u_{\varepsilon}\right)+a\left(v_{\varepsilon}, \eta-v_{\varepsilon}\right) \geq \int_{\Omega} f\left(\xi-u_{\varepsilon}\right)+\int_{\Omega} g\left(\eta-v_{\varepsilon}\right)
$$

and using the same argument as in [6], page 122, we conclude that $(u, v)$ solves $(18)$ and, by uniqueness, all the sequence $\left(u_{\varepsilon}, v_{\varepsilon}\right)$ converges to $(u, v)$.

Choosing $(\xi, v) \in \mathbb{K}_{2}$ we see that $u \in \mathbb{K}_{v}$ solves

$$
a(u, \xi-u) \geq \int_{\Omega} f(\xi-u), \quad \forall \xi \in \mathbb{K}_{v},
$$

which is the obstacle problem with lower obstacle $\psi=v$. The LewyStampacchia inequalities (see [6] or [8]) implies

$$
f \leq A u \leq f \vee A v \quad \text { a.e. in } \Omega,
$$

since by (19) and (23), $A u$ and $A v \in L^{p_{0}}(\Omega)$. On the other hand, $v \in \mathbb{K}^{u}$ solves the upper obstacle problem with $\varphi=u$ and so also

$$
g \wedge A u \leq A v \leq g \quad \text { a.e. in } \Omega .
$$

From (26) and (27), (24) follows immediately.

Proposition 2.2. The solution $(u, v)$ of (18) satisfies a.e. in $\Omega$ the system

$$
\begin{aligned}
& A u=f+\frac{g-f}{2} \chi_{\{u=v\}}, \\
& A v=g-\frac{g-f}{2} \chi_{\{u=v\}},
\end{aligned}
$$

where $\chi_{\{u=v\}}$ is the characteristic function of the coincidence set $\{x \in \Omega$ : $u(x)=v(x)\}$. 
Proof: As in [10], it is easy to see that

$$
A u=f \quad \text { and } \quad A v=g \quad \text { a.e. in }\{u>v\}
$$

and, taking $(u \pm \alpha, v \pm \alpha) \in \mathbb{K}_{2}$ for arbitrary $\alpha \in \mathcal{D}(\Omega)$, we find

$$
\langle A u+A v, \alpha\rangle=\int_{\Omega}(f+g) \alpha, \quad \forall \alpha \in \mathcal{D}(\Omega) .
$$

On the other hand, in $\{u=v\}$ we have $u_{x_{i}}=v_{x_{i}}$ a.e. and so also

$$
\zeta_{j} \equiv a_{i j} u_{x_{i}}+e_{j} u=a_{i j} v_{x_{i}}+e_{j} v \equiv \vartheta_{j} \quad \text { a.e. in }\{u=v\} .
$$

But, since $\zeta_{j}-\vartheta_{j} \in L^{1}(\Omega)$ and

$\nabla \cdot(\zeta-\vartheta)=\left(\zeta_{j}-\vartheta_{j}\right)_{x_{j}}=b_{i}(u-v)_{x_{i}}+c(u-v)-A(u-v) \in L^{1}(\Omega)$,

by Lemma 2 of [7], it follows that

$$
A u=A v \quad \text { a.e. in }\{u=v\} .
$$

Then, by (30), we also have

$$
A u=A v=\frac{f+g}{2} \quad \text { a.e. in }\{u=v\},
$$

which provides (28) and (29).

Theorem 2.2. Denoting $\left(u^{\delta}, v^{\delta}\right)$ the solution of (18) corresponding to $\left(f^{\delta}, g^{\delta}\right)$, if we suppose

$$
f^{\delta} \longrightarrow f \quad \text { and } \quad g^{\delta} \longrightarrow g \quad \text { in } L^{p_{0}}(\Omega) \quad \text { as } \delta \rightarrow 0,
$$

then

$$
u^{\delta} \longrightarrow u \quad \text { and } \quad v^{\delta} \longrightarrow v \quad \text { in } H^{1}(\Omega)-\text { strong },
$$

where $(u, v)$ solves $(18)$ with $(f, g)$. Moreover if

$$
f \neq g \quad \text { a.e. in } \Omega \text {, }
$$

then the corresponding characteristic functions satisfy, as $\delta \rightarrow 0$,

$$
\chi_{\left\{u^{\delta}=v^{\delta}\right\}} \longrightarrow \chi_{\{u=v\}} \quad \text { in } L^{q}(\Omega), \quad \forall q, 1 \leq q<\infty .
$$

Proof: The convergence (32) follows easily by general continuous dependence results, being the strong convergence (32) in the noncoercive case handled in a similar way to the proof of Theorem 7.7 of page 121 of [6].

The convergence (32) implies $A u^{\delta} \longrightarrow A u$ in $H^{-1}(\Omega)$, and using, for instance (28) for $u^{\delta}$ with $\chi^{\delta}=\chi_{\left\{u^{\delta}=v^{\delta}\right\}} \longrightarrow \chi^{*}$, we obtain, when $\delta \rightarrow 0$,

$$
A u=f+\frac{g-f}{2} \chi^{*} \quad \text { a.e. in } \Omega \text {. }
$$


Comparing with (28) for $u$, the assumption (33) yields $\chi^{*}=\chi_{\{u=v\}}$ and since $\chi^{*}$ is then a characteristic function, the conclusion follows.

Remark 2.1. If $f \geq g$ a.e. in $\Omega$, from (24) we obtain $A u=f \geq g=A v$ a.e. in $\Omega$ and, since $u \geq v$ on $\partial \Omega$, the maximum principle see ([3]) will imply $u>v$ in $\Omega$. Consequently, the condition $f<g$ at least in a set of positive measure is a necessary condition for the coincidence set $\{u=v\}$ to be non-empty, as it was observed in [10].

Remark 2.2. Arguing as in Corollary 4.5 of [10], if $\Omega$ is simply connected, $\beta_{1}>\beta_{2}$ on $\partial \Omega$ and $f \leq g$ a.e. in $\Omega$, we may conclude the open set $\{u>v\}$ is connected.

Remark 2.3. The results of Theorem 2.1 and Proposition 2.2 also apply, with convenient assumptions, to the case when

$$
A v=-\left(\frac{v_{x_{i}}}{\sqrt{1+|\nabla v|^{2}}}\right)_{x_{i}}
$$

is the minimal surface operator. When the two membranes have constant mean curvature (i.e. $f=n \Lambda_{1}$ and $g=n \Lambda_{2}$ with the mean curvature $H_{\partial \Omega}$ of $\partial \Omega$ dominating $\left.\frac{n}{n-1} \max \left\{\left|\Lambda_{1}\right|,\left|\Lambda_{2}\right|\right\}, \Lambda_{1}, \Lambda_{2} \in \mathbb{R}\right)$ in [10] it was shown the existence of a unique regular solution in $W^{2, q}(\Omega) \cap C^{1, \lambda}(\bar{\Omega}), 1 \leq q<\infty$, $0 \leq \lambda<1$ under smoothness assumptions on $\partial \Omega, \beta_{1}$ and $\beta_{2}$. An extension to $\Lambda_{i} \in L^{p}(\Omega), p>n$, supp $\Lambda_{i} \subset \Omega$, with $H_{\partial \Omega} \geq 0$ and $\left\|\Lambda_{i}\right\|_{L^{p}(\Omega)}$ small, was given in [11].

\section{The $N=3$ membranes problem}

Following the notations of the previous section, we set $u_{1}=u, u_{2}=v$, $u_{3}=w, f_{1}=f, f_{2}=g$ and $f_{3}=h$ and so (1), in the case $N=3$ corresponds to find $(u, v, w) \in \mathbb{K}_{3}, \mathbb{K}_{3}$ defined as in (3), such that

$$
\begin{aligned}
a(u, \xi-u) & +a(v, \eta-v)+a(w, \zeta-w) \\
& \geq \int_{\Omega} f(\xi-u)+\int_{\Omega} g(\eta-v)+\int_{\Omega} h(\zeta-w), \quad \forall(\xi, \eta, \zeta) \in \mathbb{K}_{3} .
\end{aligned}
$$

Using $\theta_{\varepsilon}$ defined by (20) and introducing

$$
\psi=f \vee \frac{f+g}{2} \vee \frac{f+g+h}{3}, \quad \lambda=\psi-f \quad \text { and } \quad \mu=2 \psi-(f+g),(36
$$


for each $\varepsilon>0$, we consider now the approximating system

$$
\left\{\begin{aligned}
A u_{\varepsilon}+\lambda \theta_{\varepsilon}\left(u_{\varepsilon}-v_{\varepsilon}\right) & =f \\
A v_{\varepsilon}-\lambda \theta_{\varepsilon}\left(u_{\varepsilon}-v_{\varepsilon}\right)+\mu \theta_{\varepsilon}\left(v_{\varepsilon}-w_{\varepsilon}\right) & =g \quad \text { in } \Omega \\
A w_{\varepsilon}-\mu \theta_{\varepsilon}\left(v_{\varepsilon}-w_{\varepsilon}\right) & =h
\end{aligned}\right.
$$

with Dirichlet boundary conditions $u_{\varepsilon}=\beta_{1}, v_{\varepsilon}=\beta_{2}$ and $w_{\varepsilon}=\beta_{3}$ on $\partial \Omega$. We observe that under the assumptions (14), the functions $\lambda$ and $\mu$ are nonnegative and belong to $L^{p_{0}}(\Omega)$. Similarly to Proposition 2.1 we have

Proposition 3.1. There exists a solution $\left(u_{\varepsilon}, v_{\varepsilon}, w_{\varepsilon}\right) \in\left[H^{1}(\Omega]^{3}\right.$ to $(37)$ with the given Dirichlet data, and a constant $C>0$, independent of $\varepsilon>0$, such that

$$
\left\|u_{\varepsilon}\right\|_{H^{1}(\Omega)}+\left\|v_{\varepsilon}\right\|_{H^{1}(\Omega)}+\left\|w_{\varepsilon}\right\|_{H^{1}(\Omega)} \leq C .
$$

In addition

$$
v_{\varepsilon} \leq u_{\varepsilon}+\varepsilon \quad \text { and } \quad w_{\varepsilon} \leq v_{\varepsilon}+\varepsilon \quad \text { a.e. in } \Omega .
$$

Arguing as in the proof of Theorem 2.1, and observing that the variational inequality of the three equations of (37) yields a neglectable term

$\int_{\Omega}\left\{\lambda \theta_{\varepsilon}\left(u_{\varepsilon}-v_{\varepsilon}\right)\left[(\xi-\eta)-\left(u_{\varepsilon}-v_{\varepsilon}\right)\right]+\mu \theta_{\varepsilon}\left(v_{\varepsilon}-w_{\varepsilon}\right)\left[(\eta-\zeta)-\left(v_{\varepsilon}-w_{\varepsilon}\right)\right]\right\} \geq 0$,

we easily obtain (35) from (37) by letting $\varepsilon \rightarrow 0$. Clearly (39) implies $(u, v, w) \in \mathbb{K}_{3}$ and so we may apply Lewy-Stampacchia inequalities (4) to $u \in \mathbb{K}_{v}$, (5) to $w \in \mathbb{K}^{v}$ and also (6) to $v \in \mathbb{K}_{u}^{w}$, obtaining by combination

$$
f \wedge g \leq A u \wedge g \leq A v \leq g \vee A w \leq g \vee h \quad \text { a.e. in } \Omega .
$$

In conclusion, we easily deduce the following interesting result, concluding (7) in case $N=3$ :

Theorem 3.1. The solutions to (37), as $\varepsilon \rightarrow 0$, converge

$$
\left(u_{\varepsilon}, v_{\varepsilon}, w_{\varepsilon}\right) \longrightarrow(u, v, w) \quad \text { in }\left[H^{1}(\Omega)\right]^{3} \text {-weak, }
$$

where $(u, v, w) \in \mathbb{K}_{3}$ solves uniquely (35) and satisfy

$$
\left\{\begin{aligned}
f \leq A u & \leq f \vee g \vee h \\
f \wedge g \leq A v & \leq g \vee h \quad \text { a.e. in } \Omega \\
f \wedge g \wedge h \leq A w & \leq h .
\end{aligned}\right.
$$


Proposition 3.2. The solution $(u, v, w)$ of (35) satisfies a.e. in $\Omega$ the system

$$
\begin{aligned}
& A u=f+\frac{g-f}{2} \chi_{\{u=v\}}+\frac{2 h-g-f}{6} \chi_{\{u=v=w\}} \\
& A v=g+\frac{f-g}{2} \chi_{\{u=v\}}+\frac{h-g}{2} \chi_{\{v=w\}}+\frac{2 g-f-h}{6} \chi_{\{u=v=w\}} \\
& A w=h+\frac{g-h}{2} \chi_{\{v=w\}}+\frac{2 f-g-h}{6} \chi_{\{u=v=w\}}
\end{aligned}
$$

where $\chi_{\{u=v=w\}}=\chi_{\{u=v\}} \chi_{\{v=w\}}$ denotes the characteristic function of the coincidence set $\{x \in \Omega: u(x)=v(x)=w(x)\}$.

Proof: As in the proof of Proposition 2.2 we find

$$
\langle A u+A v+A w, \alpha\rangle=\int_{\Omega}(f+g+h) \alpha, \quad \alpha \in \mathcal{D}(\Omega),
$$

and, since by (41) each $A u, A v$ and $A w$ is in $L^{p_{0}}(\Omega)$, it follows

$A u=A v=\frac{f+g}{2} \quad$ a.e. in $\{u=v\} \quad$ and $\quad A v=A w=\frac{g+h}{2} \quad$ a.e. in $\{v=w\}$.

Therefore $A u=A v=A w=\frac{f+g+h}{3} \quad$ a.e. in $\{u=v=w\}=\{u=v\} \cap\{v=w\}$ yields (42), (43) and (44) by direct computation a.e. in $\Omega$.

Theorem 3.2. Let $\left(u^{\delta}, v^{\delta}, w^{\delta}\right)$ denote the solution of (35) corresponding to $\left(f^{\delta}, g^{\delta}, h^{\delta}\right)$ such that

$$
f^{\delta} \longrightarrow f, \quad g^{\delta} \longrightarrow g \quad \text { and } \quad h^{\delta} \longrightarrow h \quad \text { in } L^{p_{0}}(\Omega) \text { as } \delta \rightarrow 0 .
$$

Then

$$
u^{\delta} \longrightarrow u, \quad v^{\delta} \longrightarrow v \quad \text { and } \quad w^{\delta} \longrightarrow w \quad \text { in } H^{1}(\Omega) \text {-strong }
$$

where $(u, v, w)$ denotes the solution of $(35)$ for $(f, g, h)$. In addition, under the nondegenerating conditions

$$
\begin{aligned}
f \neq g & \text { and } \quad g \neq h \quad \text { a.e. in } \Omega, \\
f \neq \frac{g+h}{2} \quad \text { and } & h \neq \frac{f+g}{2} \quad \text { a.e. in } \Omega,
\end{aligned}
$$

the corresponding characteristic functions, as $\delta \rightarrow 0$, satisfy, for any $1 \leq$ $q<\infty$

$$
\begin{gathered}
\chi_{\left\{u^{\delta}=v^{\delta}\right\}} \longrightarrow \chi_{\{u=v\}} \quad \text { and } \quad \chi_{\left\{v^{\delta}=w^{\delta}\right\}} \longrightarrow \chi_{\{v=w\}} \quad \text { in } L^{q}(\Omega), \\
\chi_{\left\{u^{\delta}=v^{\delta}=w^{\delta}\right\}} \longrightarrow \chi_{\{u=v=w\}} \quad \text { in } L^{q}(\Omega) .
\end{gathered}
$$


Proof: Since (46) follows by standard variational methods as in the case of Theorem 2.2, we need to show (49), from which (50) follows immediately.

Setting

$$
\chi_{1}^{\delta}=\chi_{\left\{u^{\delta}=v^{\delta}\right\}},
$$

$\chi_{2}^{\delta}=\chi_{\left\{v^{\delta}=w^{\delta}\right\}}$ and $\chi_{1,2}^{\delta}=\chi_{1}^{\delta} \chi_{2}^{\delta}=\chi_{\left\{=u^{\delta}=v^{\delta}=w^{\delta}\right\}}$, as $\delta \rightarrow 0$ we may suppose that

$\chi_{1}^{\delta} \longrightarrow \chi_{1}, \quad \chi_{2}^{\delta} \longrightarrow \chi_{2} \quad$ and $\quad \chi_{1,2}^{\delta} \longrightarrow \chi_{*} \quad$ in $L^{\infty}(\Omega)$-weak*

Taking the limit in $(42)_{\delta}$ and $(44)_{\delta}$ we obtain, a.e. in $\Omega$

$$
\left\{\begin{array}{l}
A u=f+\frac{g-f}{2} \chi_{1}+\frac{2 h-g-f}{6} \chi_{*} \\
A w=h+\frac{g-h}{2} \chi_{2}+\frac{2 f-g-h}{6} \chi_{*}
\end{array}\right.
$$

which, compared with (42) and (44) for the limit functions $u$ and $w$, implies

$$
\left\{\begin{array}{l}
\frac{g-f}{2}\left(\chi_{1}-\chi_{\{u=v\}}\right)+\frac{2 h-g-f}{6}\left(\chi_{*}-\chi_{\{u=v=w\}}\right)=0 \\
\frac{g-h}{2}\left(\chi_{2}-\chi_{\{v=w\}}\right)+\frac{2 f-g-h}{6}\left(\chi_{*}-\chi_{\{u=v=w\}}\right)=0
\end{array} \text { a.e. in } \Omega\right.
$$

Due to the assumptions (47) and (48), if we show that $\chi_{*}=\chi_{\{u=v=w\}}$ then $\chi_{1}=\chi_{\{u=v\}}$ and $\chi_{2}=\chi_{\{v=w\}}$ and (49) follows easily.

From

$$
\begin{gathered}
0 \equiv \chi_{\left\{u^{\delta}=v^{\delta}\right\}}\left(u^{\delta}-v^{\delta}\right)^{+} \longrightarrow \chi_{1}(u-v)^{+}, \\
0 \equiv \chi_{\left\{v^{\delta}=w^{\delta}\right\}}\left(v^{\delta}-w^{\delta}\right)^{+} \longrightarrow \chi_{1}(v-w)^{+},
\end{gathered}
$$

we conclude, respectively, $\chi_{1}=0$ in $\{u>v\}$ and $\chi_{2}=0$ in $\{v>w\}$. Since $0 \leq \chi_{1}^{\delta} \chi_{2}^{\delta} \leq \chi_{1}^{\delta}$ we have $0 \leq \chi_{*} \leq \chi_{1}$ and analogously $0 \leq \chi_{*} \leq \chi_{2}$. Therefore we have $\chi_{*}=0$ a.e. in $\{u>v\} \cup\{v>w\}$ and it remains to show that $\chi_{*}=1$ in $\{u=v=w\}$.

From (42) and (44) in $\{u=v=w\}$ and recalling (51),

$$
\begin{aligned}
& 0 \leq A u-f=\frac{g-f}{2}+\frac{2 h-g-f}{6}=\frac{1}{3}(g+h-2 f) \\
& 0 \geq A w-h=\frac{g-h}{2}+\frac{2 f-g-h}{6}=\frac{1}{3}(f+g-2 h)
\end{aligned}
$$

and, by the assumptions (48) we must have

$$
2 f-g-h<0 \text { and } 2 h-f-g>0 \quad \text { a.e. in }\{u=v=w\} .
$$


On the other hand, from (52),

$$
\left\{\begin{array}{l}
\frac{g-f}{2}\left(\chi_{1}-1\right)+\frac{2 h-g-f}{6}\left(\chi_{*}-1\right)=0 \\
\frac{g-h}{2}\left(\chi_{2}-1\right)+\frac{2 f-g-h}{6}\left(\chi_{*}-1\right)=0
\end{array} \quad \text { a.e. in }\{u=v=w\}(54)\right.
$$

and, using (53), if $\chi_{*}<1$ we would have $f>g$ and $g>h$ and so $2 h-f-g<$ 0 , which is absurd. Then $\chi_{*}=1$ and we conclude

$$
\chi_{*}=\chi_{\{u=v=w\}}
$$

completing the proof of the theorem.

Remark 3.1. As in Remark 2.1, $f<g$ or $g<h$, at least in a set of positive measure, are necessary conditions for the coincidence sets $\{u=v>w\}$ or $\{u>v=w\}$, respectively, to be non-empty. However, from (53),

$$
f<\frac{g+h}{2} \text { and } h>\frac{f+g}{2}
$$

at least in a set of positive measure is also a necessary condition for $\{u=$ $v=w\}$ to be non-empty.

In particular, the stronger assumption $f<g<h$ a.e. in $\Omega$ implies (47) and (48) and is also a sufficient condition for the stability of the coincidence sets.

Another important and immediate consequence of the LewyStampacchia inequalities (41) is the reduction of the regularity of the solutions to (35) to the linear theory of [3]. For instance, if $p_{0}>\frac{n}{2}$ we have that $u, v$ and $w$ are Hölder continuous in $\Omega$, or by assuming, in addition,

$$
a_{i j}, e_{j} \in C^{0,1}(\bar{\Omega}) \quad \text { and } \quad b_{i}, c \in L^{\infty}(\Omega)
$$

or the regularity of the Dirichlet data on the smoother boundary

$$
\beta_{i} \in W^{2-\frac{1}{p}, p}(\partial \Omega), i=1,2,3 \quad \text { with } \quad \partial \Omega \in C^{1,1}
$$

we obtain the following result:

Theorem 3.3. Under the assumptions (55) the solutions of the $N$ membranes problem $(N=2,3)$ are in $W_{\text {loc }}^{2, p_{0}}(\Omega)$ and in $C^{1, \alpha}(\Omega)$ with $\alpha=1-\frac{n}{p_{0}}$ if $p_{0}>n$. If also (56) holds, the regularity is global, i.e., each component is in $W^{2, p_{0}}(\Omega)$ and in $C^{1, \alpha}(\bar{\Omega})$. 
Remark 3.2. The regularity $W^{2, p}$ for the $N$-membranes problem was obtained in [2] for the case $p \geq 2$ with possibly different coercive linear operators $A_{k} v=-\left(a_{i j}^{k} v_{x_{i}}\right)_{x_{j}}$, for $k=1, \ldots, N$. Theorem 3.3 extends that regularity to the case $p_{0}>1$ if $n=2$ or $p_{0} \geq \frac{2 n}{n+2}$ if $n \geq 3$ for the same operator $A_{k}=A, \forall k=1, \ldots, N$ of the form (8).

Remark 3.3. It is interesting to observe that our results on the regularity of the solutions and on the stability of the coincidence sets still hold for nonvariational two and three membranes problems for operators of the form

$$
A v=-a_{i j} v_{x_{i} x_{j}}+b_{i} v_{x_{i}}+c
$$

with $a_{i j} \in C^{0}(\bar{\Omega}), b_{i}, c \in L^{\infty}(\Omega)$, satisfying (11) and (13) (see [8]).

The 3 -membranes nonvariational problem consists in solving the system in $\Omega$ :

$$
\begin{gathered}
u \geq v \geq w \\
A u \geq f \quad \text { and } \quad(A u-f)(v-u)=0 \\
(A v-g)(u-v) \geq 0 \quad \text { and } \quad(A v-g)(v-w) \geq 0 \\
A w \leq h \quad \text { and } \quad(A w-h)(v-w)=0
\end{gathered}
$$

with the same Dirichlet boundary conditions.

Notice that this is a system of unilateral problems, which however is not exactly of the type considered in $\S 3.4$, page 301 , of [8].

\section{Acknowledgment}

*Partially supported by Project POCTI/MAT/34471/2000 of the Portuguese FCT (Fundação para a Ciência e Tecnologia.

†With [partial] support from the FCT (Fundação para a Ciência e Tecnologia). 


\section{References}

1. Azevedo, A. \& Rodrigues, J. F. \& Santos, L.The N-membranes problem for quasilinear degenerate systemsto appear

2. Chipot, M. \& Vergara-Cafarelli, G.The N-membranes problem Appl. Math. Optim. 13 (1985), $\mathrm{n}^{\mathrm{O}} 3,231-249$

3. Gilbard, D. \& Trudinger, N. S.Elliptic partial differential equations of second order2nd edition, Springer-Verlag, Berlin, 1983

4. Kinderlehrer, D. \& Stampacchia, G.An introduction to variational inequalities and their applicationsAcademic Press, New-York-London, 1980

5. Lions, J. L.Quelques méthodes de résolution des problèmes aux limites non linéairesDunod, Gauthier-Villars, Paris, 1969

6. Rodrigues, J. F.Obstacle problems in mathematical physics North Holland, Amsterdam, 1987

7. Rodrigues, J. F.Stability remarks to the obstacle problem for the $p$-Laplacian type equations Calculus of Variations and PDE's (in press)

8. Troianiello, G. M.Elliptic differential equations and obstacle problemsPlenum Press, New York, 1987

9. Vergara-Caffarelli, G.Regolarità di un problema di disequazioni variazionali relativo a due membrane Atti Accad. Naz. Lincei Rend. Cl. Sci. Fis. Mat. Natur. (8) 50 (1971) 659-662

10. Vergara-Caffarelli, G.Variational inequalities for two surfaces of constant mean curvature Arch. Rational Mech. Anal. 56 (1974/75), 334-347

11. Vergara-Caffarelli, G.Superficie con curvatura media assegnata in $L^{p}$. Applicazioni ad un problema di disequazioni variazionali Boll. Un. Mat. Ital. (4) 8 (1973), 261-277 